\title{
Cantilever based mass sensor with hard contact readout
}

\author{
Dohn, Søren; Hansen, Ole; Boisen, Anja
}

Published in:

Applied Physics Letters

Link to article, DOI:

$10.1063 / 1.2217161$

Publication date:

2006

Document Version

Publisher's PDF, also known as Version of record

Link back to DTU Orbit

Citation (APA):

Dohn, S., Hansen, O., \& Boisen, A. (2006). Cantilever based mass sensor with hard contact readout. Applied Physics Letters, 88(26), 264104. https://doi.org/10.1063/1.2217161

\section{General rights}

Copyright and moral rights for the publications made accessible in the public portal are retained by the authors and/or other copyright owners and it is a condition of accessing publications that users recognise and abide by the legal requirements associated with these rights.

- Users may download and print one copy of any publication from the public portal for the purpose of private study or research.

- You may not further distribute the material or use it for any profit-making activity or commercial gain

- You may freely distribute the URL identifying the publication in the public portal

If you believe that this document breaches copyright please contact us providing details, and we will remove access to the work immediately and investigate your claim. 


\title{
Cantilever based mass sensor with hard contact readout
}

\author{
S. Dohn ${ }^{\text {a) }}$ \\ Department of Micro- and Nanotechnology, NanoDTU, Building 345E, DK-2800 Lyngby, Denmark \\ O. Hansen \\ Danish National Research Foundation's Center for Individual Nanoparticle Functionality (CINF), \\ Department of Micro- and Nanotechnology, NanoDTU, Building 345E, DK-2800 Lyngby, Denmark
}

\author{
A. Boisen \\ Department of Micro- and Nanotechnology, NanoDTU, Building 345E, DK-2800 Lyngby, Denmark
}

(Received 20 December 2005; accepted 12 May 2006; published online 29 June 2006)

\begin{abstract}
We present a method for microcantilever resonant frequency detection. We measure the direct current from an intermittent contact once every vibration cycle between the conducting cantilever and a counterelectrode at a low bias voltage with respect to the cantilever, while the excitation frequency and amplitude are varied. The result is an almost "digital" detection of the resonant frequency. A relative frequency resolution $\Delta f / f$ of $1 / 80000$ with high signal to noise ratio in ambient conditions is demonstrated. The detection method can be applied to portable sensor systems with very high frequency nanoelectromechanical cantilevers using simple off-chip electronics. (C) 2006 American Institute of Physics. [DOI: 10.1063/1.2217161]
\end{abstract}

Micro- and nanoelectromechanical systems (NEMS) have become important in sensing applications. ${ }^{1}$ Cantilever based mass sensing, relying on a resonant frequency shift induced by the added mass, ${ }^{2}$ has a potential for very high mass resolution as demonstrated by the reported detection of masses in the $10^{-18} \mathrm{~g}$ range. ${ }^{3}$ The resonant frequency shift can be detected using optical ${ }^{3}$ or electronic $^{4}$ detection methods. Most high-resolution systems, however, rely on optical detection.

A great challenge still at hand is to make portable sensor systems working in ambient conditions with such high sensitivity. This could probably be realized using optical detection; however, delicate alignment of the detection optics to the cantilever chip is required. Electronic detection relaxes the alignment requirements, but low signal levels and high sensitivity to stray and parasitic capacitance make integration of elaborate electronics and readout systems on the cantilever chip necessary. ${ }^{5}$

In this letter we show that it is possible to detect the resonant frequency of a cantilever based sensor by measuring the time average current flowing from an electrode to the cantilever during hard contact occurring once every cycle of the cantilever vibration. The electronic detection method provides very high resolution in ambient conditions using simple low-bandwidth off-chip electronics. Moreover, the detection method does not suffer from the common problem in electronic detection methods, where the strong actuation signals often contaminate the detected signal. Previous work on a similar design ${ }^{6}$ did not focus on the sensor applications but rather on the ability to transfer single electrons by tunneling.

The resonant frequency of the cantilever is given by $f_{0} \equiv \omega_{0} /(2 \pi)=\sqrt{K / m^{*}} /(2 \pi)$, where $K$ is the spring constant and $m^{*}$ is the effective vibrational mass of the cantilever. The cantilever mass responsivity is defined by $\mathcal{R} \equiv \partial \omega_{0} / \partial m$ $=-\omega_{0} / 2 m^{*} \approx \delta \omega_{0} / \delta m$, where $\delta \omega_{0}$ is the resonant frequency shift caused by an added mass $\delta m .^{7}$ Thus a high resonant

\footnotetext{
${ }^{a)}$ Electronic mail: sd@mic.dtu.dk
}

frequency $f_{0}$ and a low effective mass $m^{*}$ are required for a high-resolution mass sensing cantilever.

The mass resolution of the cantilever is affected by energy losses, ${ }^{8}$ as the loss mechanisms add noise. The losses are accounted for by the quality factor $Q_{d}$ defined by the ratio of energy stored in the cantilever and energy lost per cycle. Several loss mechanisms, intrinsic and extrinsic, affect the quality factor of the cantilever, ${ }^{9}$ but for microcantilevers operated in ambient conditions viscous damping from the surrounding medium is dominant resulting in quite low quality factors.

The minimum measurable change in resonant frequency, $\delta \omega_{0, \text { min }}$, is determined by thermomechanical noise, ${ }^{4}$ but in most applications the noise introduced by the measurement system, $\delta \omega_{0 \text {,sys }}$, is far larger. In order to reduce $\delta \omega_{0 \text {,sys }}$ to a minimum, it is important that the primary conversion of frequency to an electrical signal results in a large signal, then the unavoidable electronic noise becomes less important in comparison to the thermomechanical noise of the cantilever. The hard contact method we describe here is one such method, since a quite high dc level $(\sim 10 \mathrm{nA})$ is the primary electrical output signal, much higher current levels than seen in electron tunneling ${ }^{6}$ detection schemes $(\sim 1 \mathrm{pA})$. A schematic of the detection setup used for hard contact readout is shown as overlay to the actual device in Fig. 1(a).

The cantilever was operated in dynamic mode by applying an actuation voltage, $V_{A}=V_{A, \mathrm{ac}}+V_{A, \mathrm{dc}}$, consisting of an ac (HP 33120A) and dc (Keithley 2400) voltage to the actuation electrode and grounding the cantilever. A small dc (HP E3611) voltage $V_{E}$ was applied to the electrode close to the apex of the cantilever through an integrating transconductance amplifier based on a Burr Brown OPA277P $(C=1 \mathrm{pF}$ and $R=22 \mathrm{M} \Omega$ ).

At sufficiently large vibrational amplitudes, the cantilever and electrode contact once every cycle, and a unidirectional current pulse train $I_{\text {in }}(t)$, with a pulse width of $t_{c}$ and a magnitude of $I_{\text {in }}=-V_{E} / R_{C}$, is supplied to the amplifier input [Fig. 1(a)]. Here $R_{C}$ is the total resistance of the contact. At steady state, the amplifier output voltage reaches a value of 

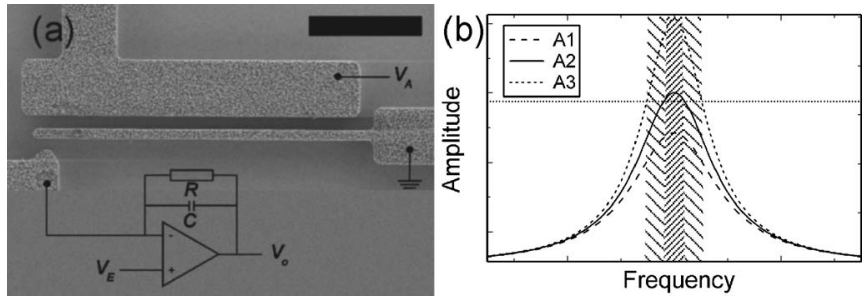

FIG. 1. The hard contact cantilever resonant frequency readout setup schematic is shown as an overlay on a SEM image of the cantilever (a). The scale bar is $20 \mu \mathrm{m}$ long. The hard contact readout principle (b). Three amplitude functions with the same quality factor but different excitation energy levels are sketched. When the amplitude exceeds a critical value (dotted line) equal to the equilibrium cantilever to electrode distance $a_{0}$, a current will flow. This occurs in a wide frequency span at high cantilever excitation energy (A3 and sparsely hatched area) and in a narrower frequency span at lower excitation energy (A2 and densely hatched area).

$V_{o, \mathrm{ss}}=V_{E}-R \overline{I_{\text {in }}}=V_{E}+\left(R / R_{C}\right)\left(t_{c} / T\right) V_{E}$, where $\overline{I_{\text {in }}}$ is the time average of the input current and $T$ is the cycle time. $V_{o, s s}$ was measured using a computer controlled Keithley 2000. It is apparent that the larger the amplitude, the larger the frequency span $\Delta f$ in which a current will flow [Fig. 1(b)]. The reciprocal relative frequency resolution $f_{0} / \Delta f$ thereby serves as a convenient measure of the quality of the measurement.

The cantilever used in the experiments was fabricated using standard microfabrication techniques. The cantilever was defined in a $2 \mu \mathrm{m}$ thick silicon dioxide film thermally grown on a silicon substrate by etching in a STS advanced oxide etcher using aluminum as etch mask. The cantilever was subsequently released by underetching using a STS reactive ion etcher for isotropic silicon etching. Then platinum was deposited onto the sides and top of the structure using e-beam evaporation. A scanning electron microscope (SEM) image of the resulting cantilever is seen in Fig. 1(a). The cantilever is $L=60 \mu \mathrm{m}$ long, $W=1.8 \mu \mathrm{m}$ wide, and $H=1.5 \mu \mathrm{m}$ thick. The theoretical resonant frequency is $390 \mathrm{kHz}$ with a mass responsivity of $\sim 1 \mathrm{kHz} / \mathrm{pg}$. The rough top surface [clearly seen in Fig. 3(a)] increases the surface area of the cantilever by roughly a factor of 10 . This is desirable for gas detection. ${ }^{10}$

For mass loading of the cantilever $\sim 1 \mu \mathrm{m}$ diameter latex spheres ${ }^{11}$ with an expected mass of $\Delta m=0.5 \mathrm{pg}$ were used. The latex-spheres were positioned on the cantilever using a manipulation setup based on a high-resolution optical Navitar microscope. A Newport XYZ stage was used for cantilever positioning, while an etched tungsten tip with a tip diameter of roughly $1 \mu \mathrm{m}$ mounted on a Burleigh PCS-5400 piezomicromanipulator was used to manipulate the latex spheres.

In all measurements $V_{A, \mathrm{ac}}=10 \mathrm{~V}_{\mathrm{pp}}$ and $V_{E}=1 \mathrm{~V}$, while $V_{A, \text { dc }}$ was varied to adjust the vibration amplitude. At a high level of the dc actuation voltage $V_{A, \mathrm{dc}} \simeq 22 \mathrm{~V}$, the resonant frequency was localized visually. $V_{o, s s}$ was measured while the excitation frequency was scanned in discrete steps near and at the resonant frequency. This was repeated at decreasing levels of $V_{A \text {,dc }}$, until the resonant peak disappeared in $V_{o, \mathrm{ss}}$. The results are shown in Fig. 2(a), which shows that the frequency span with a significant output signal, $\Delta V_{o, \text { ss }}>0.1 \mathrm{~V}$, decreases with decreasing $V_{A, \mathrm{dc}}$, is minimized at $V_{A, \mathrm{dc}}=19.2 \mathrm{~V}$, and vanishes at $V_{A, \mathrm{dc}}=19.1 \mathrm{~V}$. From the measured voltages, the time averaged current is estimated to be in the order of $\overline{I_{\text {in }}} \approx 10-50 \mathrm{nA}$.

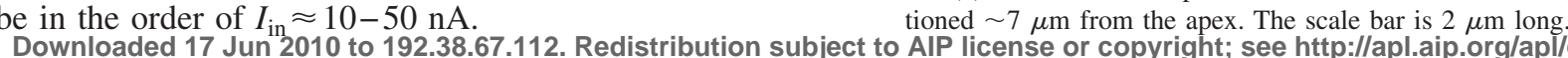

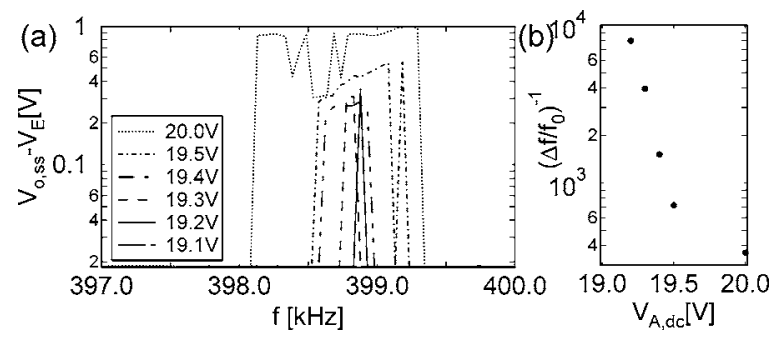

FIG. 2. The measured voltage $\Delta V_{o, s s}$ as a function of frequency at different $\mathrm{dc}$ actuation voltages (a). The reciprocal relative frequency resolution $f_{0} / \Delta f$ in the measurements (b).

In Fig. 2(b) the reciprocal relative frequency resolution $f_{0} / \Delta f$ corresponding to the measurements in Fig. 2(a) is shown. From $V_{A, \mathrm{dc}}=20 \mathrm{~V}$ to $V_{A, \mathrm{dc}}=19.2 \mathrm{~V}$, the reciprocal relative frequency resolution increases from $f_{0} / \Delta f=300$ to $f_{0} / \Delta f \simeq 8000$. Note that at $V_{A, \mathrm{dc}}=19.2 \mathrm{~V}$ the resolution is limited by the resolution in applied frequencies.

A demonstration of the detection method used for mass sensing is shown in Fig. 3. Here two latex spheres were placed on the cantilever one after the other; the SEM image in Fig. 3(a) shows the apex of the cantilever with both latex spheres in place. The latex spheres were deformed by the manipulation but remained intact. The frequency responses with no, one, and two latex spheres are shown in Fig. 3(b). The change in resonant frequency for each added latex sphere was $\sim-1.1 \mathrm{kHz}$, and the reciprocal relative frequency resolution was $20000<f_{0} / \Delta f \leqslant 80000$. The change in resonant frequency is more than twice as large as anticipated. The reason for the large frequency shift could be a lower effective mass density of the cantilever due to the very rough surface with rather deep holes etched into the cantilever material, but the latex spheres could also have a higher mass than expected due to absorbed water and salts from the atmosphere and the shipping liquid.

In all microelectromechanical system (MEMS) or NEMS systems where contact between surfaces is required or unavoidable, sticking is a serious concern. The nonstick condition for the detection method here is that the elastic energy stored in the cantilever with Young's modulus $Y$, deflected a distance $a_{0}$, exceeds the adhesive energy $A_{C} E_{\text {adh }}$, due to a contact of area $A_{C}$. $E_{\text {adh }}$ depends strongly on the mechanisms involved in the adhesion, but in worst case, where chemical bonds are formed, the order of magnitude is $E_{\text {adh }} \sim 1 \mathrm{~J} / \mathrm{m}^{2}{ }^{12}$ These considerations lead to the geometrical design rule $(W / L)^{3} H a_{0}^{2} / A_{C}>8 E_{\text {adh }} / Y$ that must be fulfilled to unconditionally avoid sticking. Assuming a blunt wedgelike electrode and thereby a contact area of $A_{C}=H \ell$, the

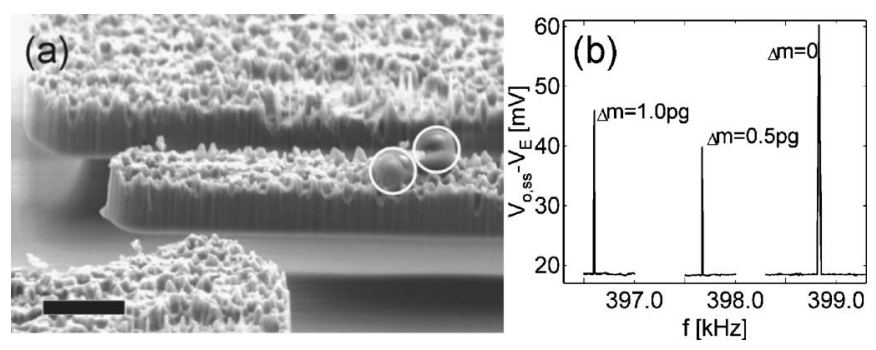

FIG. 3. SEM image of the cantilever apex with two latex spheres added (a). The frequency response with no, one, and two latex spheres on the cantilever (b). The two latex spheres are marked with white rings and are posi- 
length $\ell$ of the contact region in the experimental devices presented here must fulfil $\ell<1 \mu \mathrm{m}$. This is in perfect agreement with observations, since sticking was never observed in these devices. For linear geometrically scaled devices, $\ell$ scales linearly, which should not pose problems, because the better lithographic tools required also improve the wedge sharpness.

The average current measured in this method depends on the ratio of contact time to cycle time and on the contact resistance. These parameters are thus important when considering the scaling behavior of the method. Assuming that $N$ coherent electron channels are formed in the contact, the contact resistance becomes $R_{C}=h /\left(2 e^{2} N\right) \approx 13 \mathrm{k} \Omega / \mathrm{N},{ }^{13}$ where $h$ is Planck's constant and $e$ is the unit charge. Note that other resistance contributions, such as bulk and spreading resistances, are unimportant if low resistivity $(\sim 2 \mu \Omega \mathrm{cm})$ metals are used. The number of electron channels is probably affected by scaling, and in an ultimate scaled device a single channel is assumed to remain, which still results in a useful low resistance.

To a first approximation, the contact time is determined by the cantilever beam dynamics. During the contact time $t_{c}$, the cantilever motion is described by a superposition of modes for the cantilever pinned to the end deflection $a_{0}$. It follows that the contact time $t_{c}$ scales with geometry and material parameters exactly as the cycle time $T$ does; as a result the ratio of these is unaffected by geometrical scaling.

Compared to other electronic readout methods, the main advantage of the hard contact method is that a dc current is measured. Therefore simple low-bandwidth off-chip electronics can be used, and unavoidable parasitic and stray capacitances do not affect the frequency resolution; hence scaling to very high resonant frequency NEMS cantilevers is straightforward. Since delicate alignment of the cantilever chip to the detection system is not required, system integration is facilitated, especially for portable systems where the cantilever chip is a consumable; in such applications, this method may compete favorably with optical detection methods. A portable system based on the hard contact method combined with readout at higher bending modes ${ }^{14}$ operated in ambient conditions could prove very sensitive and economical.

We have described a method for detection of the resonant frequency of microcantilevers based on measurement of a direct current from an intermittent hard contact to a biased electrode. The method was shown experimentally to provide high quality resonant frequency detection, since a relative frequency resolution of 1/80 000 was demonstrated in ambient conditions at a signal to noise ratio of several hundreds. We have shown that the method is scalable to high resonant frequency NEMS applications and provided a design rule to prevent sticking. Thus we are convinced that the method will prove useful in many cantilever based sensor systems, in particular, in portable systems, where it could prove to be a useful alternative to optical sensing as no critical alignment to the detection circuitry is needed with the method.

CINF is sponsored by The Danish National Research Foundation.

${ }^{1}$ M. Roukes, Phys. World 14, 25 (2001).

${ }^{2}$ G. Y. Chen, T. Thundat, E. A. Wachter, and R. J. Warmack, J. Appl. Phys. 77, 3618 (1995).

${ }^{3}$ T. Ono, X. X. Li, H. Miyashita, and M. Esashi, Rev. Sci. Instrum. 74, 1240 (2003).

${ }^{4}$ K. L. Ekinci, X. M. H. Huang, and M. L. Roukes, Appl. Phys. Lett. 84, 4469 (2004).

${ }^{5}$ E. Forsen, G. Abadal, S. Ghatnekar-Nilsson, J. Teva, J. Verd, R. Sandberg, W. Svendsen, F. Perez-Murano, J. Esteve, E. Figueras, F. Campabadal, L. Montelius, N. Barniol, and A. Boisen, Appl. Phys. Lett. 87, 43507 (2005).

${ }^{6}$ A. Erbe, R. H. Blick, A. Tilke, A. Kriele, and J. P. Kotthaus, Appl. Phys. Lett. 73, 3751 (1998).

${ }^{7}$ K. L. Ekinci, Y. T. Yang, and M. L. Roukes, J. Appl. Phys. 95, 2682 (2004).

${ }^{8}$ G. Stemme, J. Micromech. Microeng. 1, 113 (1991).

${ }^{9}$ K. Y. Yasumura, T. D. Stowe, E. M. Chow, T. Pfafman, T. W. Kenny, B. C. Stipe, and D. Rugar, J. Microelectromech. Syst. 9, 117 (2000).

${ }^{10}$ A. G. Hansen, M. W. Mortensen, E. T. Andersen, J. Ulstrup, A. Kühle, J. Garnæs, and A. Boisen, Probe Microsc. 2, 139 (2001).

${ }^{11}$ Kisker, pps-1.0 polystyrene micropartikel, $992 \mathrm{~nm}$.

${ }^{12}$ W. P. Maszara, G. Goetz, A. Cavaglia, and J. B. McKetterick, J. Appl. Phys. 64, 4943 (1988).

${ }^{13}$ S. Datta, Electronic Transport In Mesoscopic Systems (Cambridge University Press, Cambridge, 1995), p. 57.

${ }^{14}$ S. Dohn, R. Sandberg, W. Svendsen, and A. Boisen, Appl. Phys. Lett. 86, 233501 (2005) 